\title{
Labrune Syndrome: A Very Rare Association of Leukoencephalopathy, Cerebral Calcifications, and Cysts
}

\author{
Anagha R. Joshi ${ }^{1}$ Kiran Kulkarni ${ }^{2} \quad$ Ankita U. Shah ${ }^{1}$ \\ ${ }^{1}$ Department of Radiology, Lokmanya Tilak Municipal Medical College \\ and General Hospital, Mumbai, Maharashtra, India \\ 2 Department of Radiology, NMR Scan Centre, Hubli, Karnataka, India \\ Address for correspondence Anagha R. Joshi, MD, Department of \\ Radiology, LTMMC and LTMGH, Sion, Mumbai, Maharashtra, India \\ (e-mail: anaghajoshi2405@gmail.com).
}

Indian J Radiol Imaging 2021;31:772-775.

\begin{abstract}
Leukoencephalopathy, cerebral calcifications, and cysts (LCC) form a very rare association which is named as "Labrune syndrome" after Labrune who reported the first case in 1996. To the best of our knowledge only eight to 10 cases have been reported in literature to date. We report a case of a 26-year-old male with onset of neurological symptoms in late adolescence (at 19 years of age) and presented with complains of continued seizures for 7 years, giddiness with imbalance, and slowly progressive motor

Keywords

- Labrune syndrome

- leukoencephalopathy

- cerebral calcifications

- cysts symptoms. MRI brain revealed multiple calcifications in bilateral basal ganglia, cerebral white matter, multiple cystic lesions in the supratentorial white matter, and abnormal diffused bilateral white matter T2 hyperintensity suggesting leukoencephalopathy. Histopathological evaluation revealed prominent congested blood vessels suggestive of angiomatous changes and cystic areas suggestive of secondary gliosis.
\end{abstract}

\section{Introduction}

Leukoencephalopathy, cerebral calcifications, and cysts (LCC) form a very rare association which is termed "Labrune syndrome" after Labrune who reported the first case in $1996 .{ }^{1}$ Clinical features include repeated seizures, gradual reduction of cognition, pyramidal, and extrapyramidal symptoms. CT features are extensive calcifications involving bilateral basal ganglia and bilateral deep cerebellar nuclei. MRI demonstrates extensive abnormal white matter hyperintensity suggesting leukoencephalopathy. Development of large parenchymal cysts causes complications like compression and pressure effect on surrounding structures necessitating surgical treatment. ${ }^{1,2}$ Histopathology shows mainly angiomatous changes and cystic gliotic areas. ${ }^{1}$ We report a case of LCC and discuss the findings with review of literature.

\section{Case Report}

A 26-year-old male patient with complaints of generalized tonic-clonic seizures, giddiness with imbalance, and slowly progressive motor symptoms for 7 years was referred to us for MRI brain. General physical, ophthalmologic, and neurological examinations did not reveal any significant abnormality. Initially the neurological examination revealed reduced muscle power (four-fifth) on the right side with mild spasticity which was progressive. Complete blood count, liver and renal function tests, sedimentation rate, calcium, phosphate, alkaline phosphatase, lactate levels, serum thyroid, and parathyroid hormones revealed no abnormality. Cytomegalovirus, toxoplasma gondii, hydatid cyst, HIV 1, and 2 serological tests were all negative. No significant abnormality was seen on cervical, thoracic, and abdominal CT examinations.
DOI https://doi.org/ $10.1055 / \mathrm{s}-0041-1736398$ ISSN 0971-3026. (c) 2021. Indian Radiological Association. All rights reserved.

This is an open access article published by Thieme under the terms of the Creative Commons Attribution-NonDerivative-NonCommercial-License, permitting copying and reproduction so long as the original work is given appropriate credit. Contents may not be used for commercial purposes, or adapted, remixed, transformed or built upon. (https://creativecommons.org/ licenses/by-nc-nd/4.0/)

Thieme Medical and Scientific Publishers Pvt. Ltd., A-12, 2nd Floor, Sector 2, Noida-201301 UP, India 

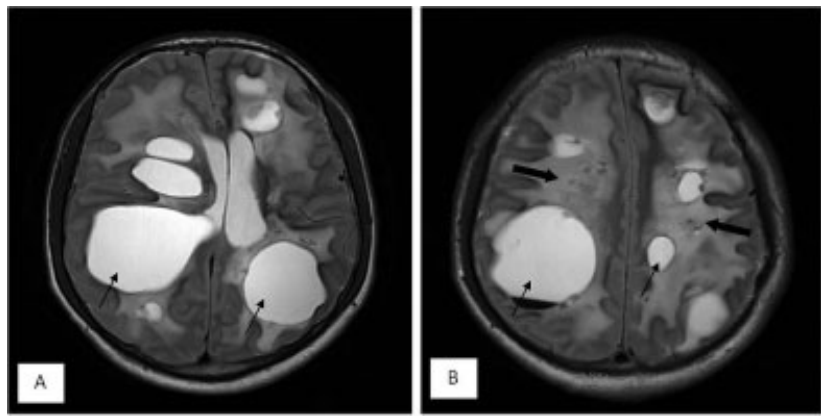

Fig. 1 ( $A$ and B) T2 W MRI axial images show the multiple cystic lesions (thin black arrows), diffuse white matter hyperintense signal in white matter (thick black arrows) of bilateral cerebral hemispheres.
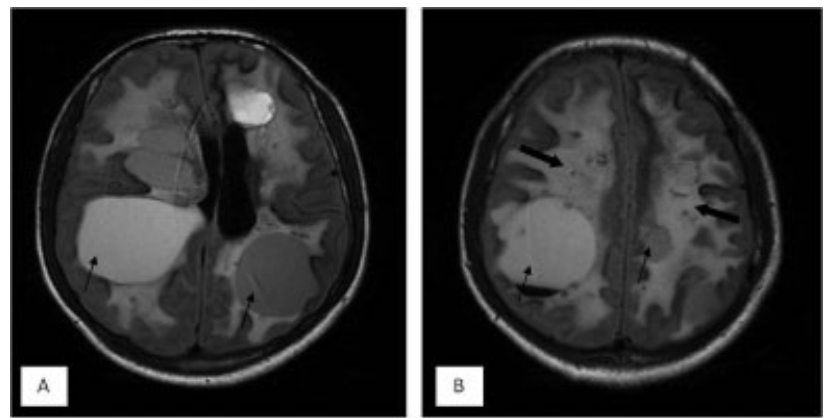

Fig. 2 (A and B) FLAIR MRI axial images show the multiple cystic lesions (thin black arrows), diffuse white matter hyperintense signal in white matter (thick black arrows) of bilateral cerebral hemispheres.

MRI of brain revealed multiple varying sized round to oval cystic lesions appearing uniformly hyperintense to CSF on T2, variably hyperintense to CSF on FLAIR, and uniformly hypointense on T1 scattered throughout the white matter of bilateral cerebral hemispheres (-Figs. 1,2,3). These lesions did not show restriction of diffusion and showed peripheral post-contrast enhancement. The cyst in the high parietal region showed a small area of blood-fluid level. These lesions caused a mass effect in the form of compression of the frontal horn and body of right lateral ventricle, third ventricle and bilateral cerebral peduncles and mild midline shift to left. There was extensive T2 and FLAIR hyperintensity in the subcortical and deep white matter of bilateral cerebral hemispheres. There were extensive calcifications involving
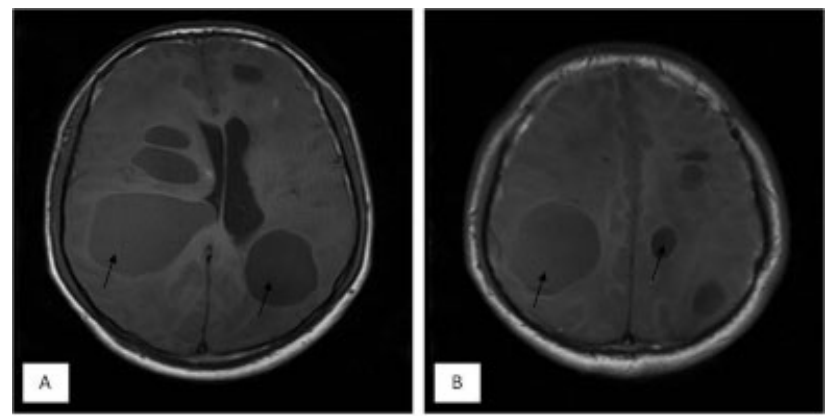

Fig. 3 (A and B) T1 W MRI axial images show the multiple cystic lesions (thin black arrows) in the bilateral cerebral hemispheres.
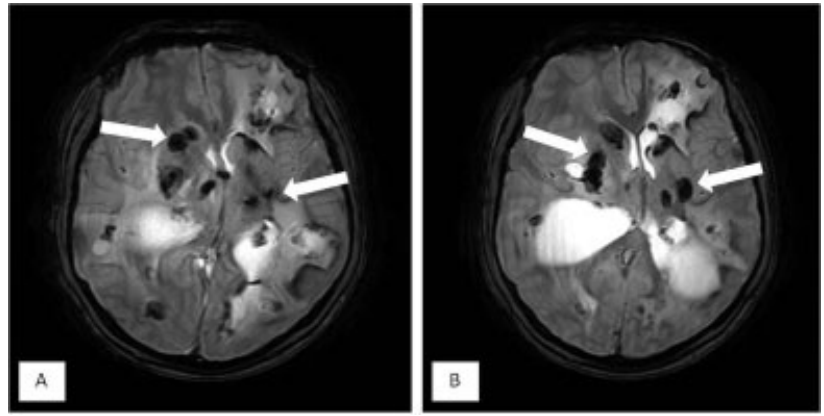

Fig. 4 ( $A$ and B) T2 FFE GRE axial images show the multiple calcifications (white arrows) in the bilateral caudate, putamina, thalami, right cerebral peduncle, and bilateral cerebral white matter.

bilateral caudate nuclei, putamina, thalami, right cerebral peduncle (-Fig. 4).

The patient had undergone multiple serial MRI brain examinations and a single CT brain from the onset of the clinical symptoms at the age of 19 years. On comparing with those previous studies there was a significant increase in the number, size, and distribution of the cerebral cystic lesions with increase in the mass effect on surrounding structures. Also, there was an increase in the extensive T2 and FLAIR hyperintensity in the subcortical and deep white matter of bilateral cerebral hemispheres which was suggestive of leukoencephalopathy. The calcifications also increased in size and distribution.

\section{Discussion}

The combination of cerebral cysts, calcifications, perilesional white matter edema generally directs to the consideration of a parasitic infection, especially, cysticercosis or echinococcus alveolaris infection. Nonetheless no serologic or histopathologic confirmatory evidence was found. Thus, considering the rare association of cerebral cysts, basal ganglia calcifications, and supratentorial cysts, we began with literature search. Our case resembled exactly with LCC previously described by Labrune et $\mathrm{al}^{1}$ and Nagae-Poetscher ${ }^{2}$ et al. The main imaging features of LCC were extensive brain calcifications, leukodystrophy, and formation of parenchymal cysts. ${ }^{1}$ The main symptoms and signs are cognitive decline, convulsive seizure, pyramidal, extrapyramidal, and/or cerebellar signs. The patients present with the clinical features generally from early infancy to adolescence. Our patient presented with his first generalized seizure at 19 years of age. Initially his seizures were rare and responded well to anticonvulsant drug therapy. The pyramidal symptoms developed 4 to 5 years after the first convulsive seizure. Six years later, he had slowly progressive motor symptoms in the form of right-sided weakness and slurring of speech. This slow evolution of the disease in our patient is in line with the very slow progression rate described for LCC.

The clinical course of LCC is mild, however, a serious and continuous degeneration of the nervous system was shown on neuroimaging. Increased white matter signal intensity relatively sparing the $\mathrm{U}$ fibers and corpus callosum, cerebral cysts, extensive coarse calcifications in the basal ganglia, brain stem, 
and subcortical white matter in our case were similar to those of other cases described in the literature. During the course of the 7 years many of the cysts enlarged gradually and caused mass effect and compression of the close structures such as ventricles and cerebral peduncles. The increase in the size of the cysts may be the effect of continuous increase in fluid content of the white matter. ${ }^{1,2}$ Similar circumstance was also seen in other conditions, such as leukoencephalopathies and some intracranial tumors. ${ }^{3-5}$ Coalition of the microcysts containing edema fluid could be a probable mechanism for the increase in size of cysts. The expansion of cysts in our case may be the result of such a mechanism.

The main differentials considered were hydatid, cysticercosis, and cryptococcosis. Neurocysticercosis would present with multiple cystic lesions with variable parenchymal calcifications. ${ }^{6}$ HIV patients with cryptococcosis present with parenchymal calcifications and gelatinous pseudocysts. ${ }^{7}$ Nonetheless no serologic or histopathologic confirmatory evidence was found. Also, leukoencephalopathy has not been described with these entities. Fahr's disease presents with extensive basal ganglia and cerebellar nuclei calcifications. Nonetheless the other findings of LCC are not the features of Fahr's disease. Children who receive chemotherapy and radiotherapy for malignancies also show features of basal ganglia calcifications with white matter changes. Coat's disease in association with leukoencephalopathy, cysts, and calcification has been called as "Coat's plus." ${ }^{8}$ Unilateral retinal telangiectasia with exudation commonly occurring in boys sporadically without systemic features is referred to as Coat's disease. Bilateral retinal telangiectasia with exudation along with systemic features in the form of LCC is referred to as "Coat's plus." The ophthalmologic examination in our case was normal, thus ruling out the possibility of "Coat's plus." Cerebroretinal microangiopathy with calcification and cysts (CRMCC) incorporates both LCC and Coat's plus diseases. ${ }^{9}$

There is a controversy surrounding the etiopathogenesis of LCC. The basic abnormality found in histopathological examination was obliterative microangiopathy. ${ }^{1}$ It is hypothesized that the necrotic process secondary to obliterative

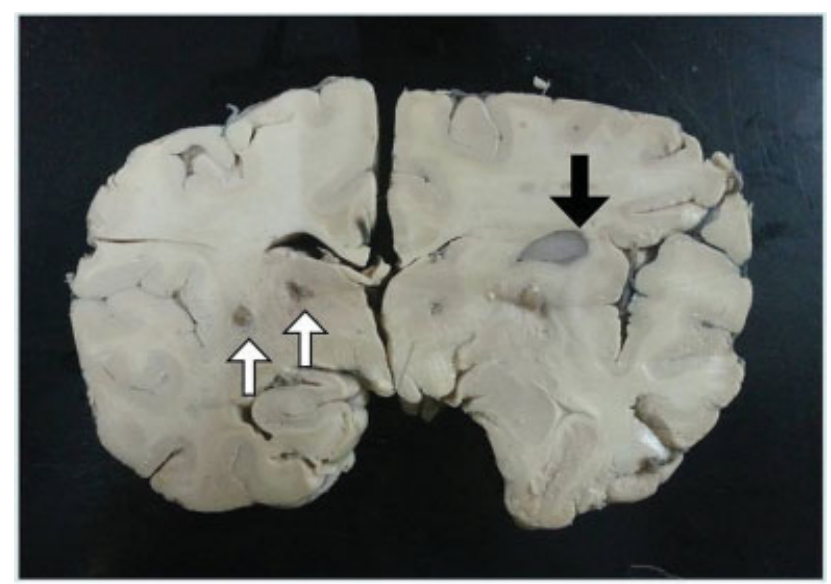

Fig. 5 Gross autopsy specimen reveals variable sized cysts (black arrow) predominantly in white matter and few in subcortical area with few foci of calcification (white arrows) with focally congested vessels in the leptomeninges.

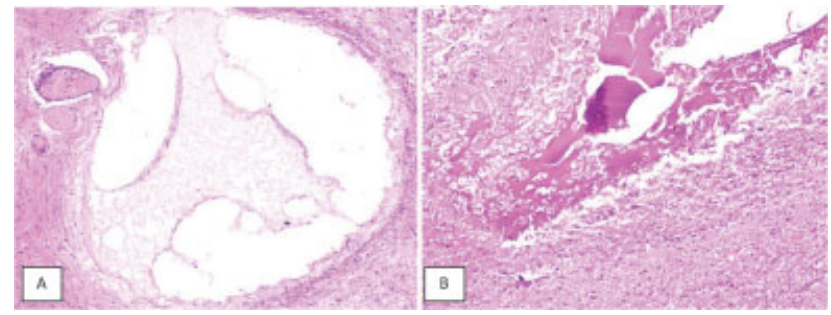

Fig. 6 (A and B) Reveals multiple variable sized cysts with gelatinous eosinophilic contents.

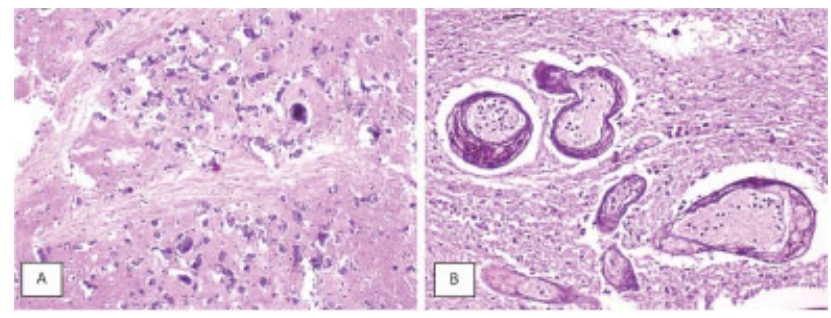

Fig. 7 ( $\mathrm{A}$ and $\mathrm{B}$ ) Reveals dystrophic calcification in the cerebral parenchyma and vessel wall.

microangiopathy is the cause for cyst formation and calcification is the result of dystrophic calcification. White matter abnormal hyperintense signal is due to changes in water content and not due to myelination abnormality.

Gross pathology specimens revealed external cerebral and cerebellar parenchymal moderate edema. Leptomeninges were thin and shiny with focally congested vessels. Serial cut sections revealed multiple variable sized cysts predominantly in white matter and few in subcortical area with few foci of calcification. These cysts were filled with mucoid gelatinous material (-Fig. 5).

Edematous changes in the brain parenchyma, exaggerated proliferation of abnormal small vessels, significant gliosis, and micro calcifications were the chief histopathological findings reported by Labrune et al in two of three patients of Labrune syndrome. The chief histopathological findings in our specimen were angiomatous changes in the form of abnormal prominent congested vessels, multiple cystic areas with gelatinous eosinophilic contents resembling plasma, foci of dystrophic calcification in the cerebral parenchyma and vessel wall, many foci of gliosis, Rosenthal fibers and histiocytic aggregates with cholesterol clefts. These findings are consistent with LCC (-Figs. 6-8).

In conclusion, although there is definitive imaging association in the form of the triad in LCC, the exact etiology of the triad remains unknown. Nonetheless LCC represents a specific

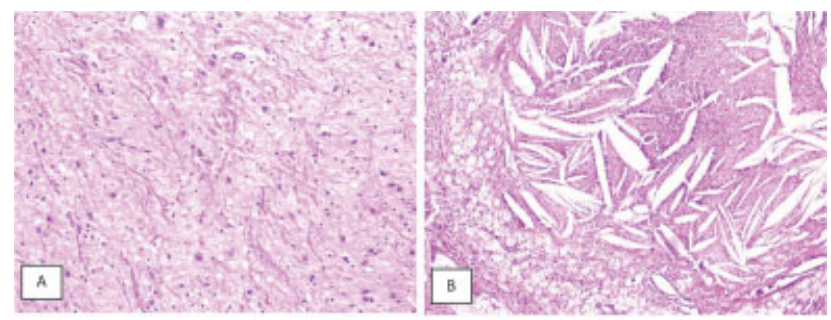

Fig. 8 (A and B) Reveals foci of gliosis, Rosenthal fibers, and histiocytic aggregates with cholesterol clefts. 
recognizable clinic-radiologic entity. The most noticeable histopathologic feature is cerebral angiomatous changes. In spite of these relatively characteristic imaging and histopathologic findings, the clinical features are not uniform.

Thus, it may be contemplated that the older onset age, normal intelligence, and very slow progression, as in our patient, may indicate the presence of the adult form of this rare disease.

\section{Financial Support and Sponsorship}

None.

Source(s) of Support in the Form of Grants, Equipment, Drugs, or All of These

None.

\section{Conflict of Interest}

None declared.

\section{Acknowledgment}

None.

\section{References}

1 Labrune P, Lacroix C, Goutières F, et al. Extensive brain calcifications, leukodystrophy, and formation of parenchymal cysts: a new progressive disorder due to diffuse cerebral microangiopathy. Neurology 1996;46(05):1297-1301

2 Nagae-Poetscher LM, Bibat G, Philippart M, et al. Leukoencephalopathy, cerebral calcifications, and cysts: new observations. Neurology 2004;62(07):1206-1209

3 Ben-Zeev B, Gross V, Kushnir T, et al. Vacuolating megalencephalic leukoencephalopathy in 12 Israeli patients. J Child Neurol 2001; 16(02):93-99

4 Pridmore CL, Baraitser M, Harding B, Boyd SG, Kendall B, Brett EM. Alexander's disease: clues to diagnosis. J Child Neurol 1993;8(02): 134-144

5 Lohle PN, van Mameren H, Zwinderman KH, Teepen HL, Go KG, Wilmink JT. On the pathogenesis of brain tumour cysts: a volumetric study of tumour, oedema and cyst. Neuroradiology 2000; 42(09):639-642

6 Turkulov V, Madle-Samardzija N, Canak G, Vukadinov J, AleksićDordević M Clinical and diagnostic approaches to neurocysticercosis. Med Pregl 2001;54(7-8):353-356

7 Caldemeyer KS, Mathews VP, Edwards-Brown MK, Smith RR. Central nervous system cryptococcosis: parenchymal calcification and large gelatinous pseudocysts. AJNR Am J Neuroradiol 1997;18(01):107-109

8 Corboy JR, Gault J, Kleinschmidt-DeMasters BK. An adult case of leukoencephalopathy with intracranial calcifications and cysts. Neurology 2006;67(10):1890-1892

9 Briggs TA, Abdel-Salam GM, Balicki M, et al. Cerebroretinal microangiopathy with calcifications and cysts (CRMCC). Am J Med Genet A 2008;146A(02):182-190 